\title{
Multiobjective Robust Control with HIFOO 2.0 *
}

\author{
Suat Gumussoy ${ }^{*}$ Didier Henrion ${ }^{* *}$ Marc Millstone ${ }^{* * *}$ \\ Michael L. Overton ${ }^{* * * *}$ \\ * Katholieke Universiteit Leuven, Department of Computer Science, \\ Belgium \\ suat. gumussoy@cs . kuleuven . be \\ ** LAAS-CNRS, University of Toulouse, France, and Faculty of \\ Electrical Engineering, Czech Technical University in Prague, Czech \\ Republic \\ henrion@laas.fr \\ *** Courant Institute of Mathematical Sciences, New York University, \\ $U S A$ \\ millstone@cims.nyu.edu \\ **** Courant Institute of Mathematical Sciences, New York University, \\ $U S A$ \\ overton@cims.nyu.edu
}

\begin{abstract}
Multiobjective control design is known to be a difficult problem both in theory and practice. Our approach is to search for locally optimal solutions of a nonsmooth optimization problem that is built to incorporate minimization objectives and constraints for multiple plants. We report on the success of this approach using our public-domain MATLAB toolbox HIFOO 2.0, comparing our results with benchmarks in the literature.
\end{abstract}

Keywords: robust control; multiobjective control; optimization

\section{INTRODUCTION}

Multiobjective control aims at designing a feedback control law meeting potentially conflicting specifications defined on various input/output channels.

In the context of linear systems, a standard approach to multiobjective control is the Lyapunov shaping paradigm proposed in the mid 1990s by Scherer et al. [1997], as an outgrowth of the LMI (linear matrix inequality) formalism of Boyd et al. [1994]. Within this scope, multiobjective controller design boils down to semidefinite programming (linear programming over the cone of positive semidefinite matrices) provided all the closed-loop specifications are certified simultaneously by a unique quadratic Lyapunov function. Moreover, the controller is retrieved a posteriori via tedious linear algebra, and its order is equal to the order of the open-loop plant plus the order of the weighting functions, which can be quite high in practice, in contradiction with simplicity of implementability requirements of embedded control laws. Another computational approach

\footnotetext{
* The research of S. Gumussoy was supported by the Belgian Programme on Interuniversity Poles of Attraction of OPTEC, the Optimization in Engineering Centre of the K.U.Leuven. The research of D. Henrion was partly supported by project MSM6840770038 of the Ministry of Education of the Czech Republic. The work of M. Millstone and M.L. Overton was partly supported by the U.S. National Science Foundation under grants DMS-0602235 and DMS0714321; views expressed in the paper are those of the authors and not of the NSF. The work of M.L. Overton was also partly funded by Université Paul Sabatier, Toulouse, France.
}

to multiobjective control exploits the parametrization of all stabilizing controllers described e.g. by Vidyasagar [1985]. Linear programming can be used in this context to design controllers, see e.g. Boyd and Barratt [1991], but they are typically of very high order.

Following a decade of research efforts, these restrictions have been gradually relaxed (distinct Lyapunov functions for distinct performance channels, parameter-dependent Lyapunov functions, decoupling between Lyapunov and controller variables, lower-order controller design) at the price of an increased computational burden. The ROMULOC (robust multiobjective control) toolbox is a recent public-domain MATLAB implementation of these techniques, see Peaucelle and Arzelier [2006].

Particular cases of multiobjective robust control problems include strong stabilization (where a plant must be stabilized by a controller which is itself stable, see Vidyasagar [1985]) or simultaneous stabilization (where a single controller must be found that stabilizes several plants, see Blondel [1994]). Most of the algorithms or heuristics available to solve these problems also typically result in very high order controllers.

In this paper, we introduce the new release 2.0 of our freely available package HIFOO, which is aimed at removing the above mentioned limitations in the context of multiobjective controller design. First, the controller order is fixed at the outset, allowing for lower-order controller design. Second, no Lyapunov or lifting variables 
are introduced to deal with the conflicting specifications. The resulting optimization problem is formulated on the controller coefficients only, resulting in a typically smalldimensional, nonsmooth, nonconvex optimization problem that does not require the solution of any large convex subproblems, relieving the computational burden typical of Lyapunov LMI techniques. Because finding the global minimum of this optimization problem may be hard, we use an algorithm that searches only for local minima. While no guarantee can be made about the behaviour of this algorithm, in practice it is often possible to determine an acceptable controller quite efficiently.

See also Apkarian et al. [2008] for nonsmooth nonconvex optimization techniques applied to multiobjective robust control. As far as we know, no software implementation of these techniques is publicly available at present.

\section{PROBLEM FORMULATION}

The $i$ th generalized plant $P^{i}=\left(A^{i}, B^{i}, C^{i}, D^{i}\right)$ describes the state-space equations

$$
\begin{aligned}
& \dot{x}^{i}(t)=A^{i} x^{i}(t)+B_{1}^{i} w^{i}(t)+B_{2}^{i} u^{i}(t), \\
& z^{i}(t)=C_{1}^{i} x^{i}(t)+D_{11}^{i} w^{i}(t)+D_{12}^{i} u^{i}(t), \\
& y^{i}(t)=C_{2}^{i} x^{i}(t)+D_{21}^{i} w^{i}(t)+D_{22}^{i} u^{i}(t),
\end{aligned}
$$

where $A^{i} \in \mathbb{R}^{n^{i} \times n^{i}}, D_{12}^{i} \in \mathbb{R}^{p_{1}^{i} \times m_{2}}, D_{21}^{i} \in \mathbb{R}^{p_{2} \times m_{1}^{i}}$, with other matrices having compatible dimensions. The signals $\left(z^{i}, w^{i}, y^{i}, u^{i}\right)$ respectively represent the regulated outputs, the exogenous inputs (including disturbance and commands), the measured (or sensor) inputs, and the control inputs. Let $N$ be the number of plants.

The problem is to choose a single controller

$$
K=\left(A_{K}, B_{K}, C_{K}, D_{K}\right)
$$

with state-space equations

$$
\begin{aligned}
\dot{x}_{K}(t) & =A_{K} x_{K}(t)+B_{K} y(t), \\
u(t) & =C_{K} x_{K}(t)+D_{K} y(t),
\end{aligned}
$$

where $A_{K} \in \mathbb{R}^{n_{K} \times n_{K}}$, with $B_{K}, C_{K}, D_{K}$ having dimensions that are compatible with $A_{K}$ and the generalized plant matrices. The controller order $n_{K}$ is fixed, so it can be specified by the designer. The $\mathcal{H}_{\infty}$ norm of the $i$ th closed loop system is the norm of the transfer function from input $w^{i}$ to output $z^{i}$; see Zhou et al. [1996] for details. The complex stability radius is a useful alternative measure when no $w^{i}$ and $z^{i}$ performance channels are specified and hence the $\mathcal{H}_{\infty}$ norm is not defined: for a stable closed loop system, this is the largest 2-norm perturbation to the closed loop system that can be tolerated while guaranteeing that the perturbed system remains stable. The spectral abscissa of a closed loop system is the largest of the real parts of its poles (eigenvalues).

Let $\beta_{j}, j=1, \ldots, N$ each be a real number or $\infty$, and consider the following optimization problem:

$$
\min _{K} \max _{j=1, \ldots, N}\left\{g_{j}(K): \beta_{j}=\infty\right\}
$$

subject to $g_{j}(K) \leq \beta_{j}, \quad j=1,2, \ldots, N$, where each $g_{j}$ is one of the following supported functions of the closed-loop system for $P^{j}$, abbreviated by a single letter as follows:

- ' $\mathrm{h}$ ': $\mathcal{H}_{\infty}$ norm ( $\infty$ if unstable);

- 'r': reciprocal of complex stability radius $(\infty$ if unstable);

- 's': spectral abscissa.

The functions $g_{j}$ are all nonconvex, nonsmooth functions of the controller matrices. Thus, the optimization problem is potentially quite difficult. We focus on two scenarios.

Scenario 1. All $\beta_{j}=\infty$, and all $g_{j}$ are 's'. Thus the problem is to minimize the maximum of the real parts of the closed loop poles of all plants. This approach is suitable for simultaneous stabilization, as the goal is to move the poles as far left in the complex plane as possible (in the minmax sense). If the final objective value is negative, all closed loop plants are stable.

Scenario 2. All $\beta_{j}=\infty$, and all $g_{j}$ are ' $\mathrm{h}$ '. The problem is to stabilize all plants and minimize the maximum of the $\mathcal{H}_{\infty}$ norms of the closed loop plants. The advantage over Scenario 1 is that the goal is to not only stabilize the plants, but also optimize their $\mathcal{H}_{\infty}$ performance (in the minmax sense). The disadvantage is that evaluating the $\mathcal{H}_{\infty}$ norm repeatedly is more time-consuming than computing the spectral abssissa repeatedly, and I/O performance channels must be specified.

These two scenarios are the ones for which benchmarking is done in Section 5. However, the interface to HIFOO is sufficiently flexible that there are many other alternative ways to call it which may be of interest to users. For example:

Alternative 3. All $\beta_{j}=\infty$, and all $g_{j}$ are ' $r$ '. The problem is to stabilize all plants and maximize the minimum of the complex stability radii of the closed loop plants (minimize the maximum of their reciprocals). The advantage is that the complex stability radius is a more robust measure of stability than the spectral abscissa, so this is an appropriate alternative to Scenario 2 when I/O performance channels are not specified.

Alternative 4. This is a more specific example. Suppose $\beta_{1}=\infty, \beta_{2}=100, g_{1}$ is ' $\mathrm{h}$ ' and $g_{2}$ is ' $r$ '. The problem is to stabilize both plants and minimize the $\mathcal{H}_{\infty}$ norm of the first closed loop plant subject to the complex stability radius of the second being at least 0.01 .

If one wishes to impose restrictions, such as stability, on the controller (so-called strong stabilization), it is possible to do so using the multiple-plant model above by defining a plant $P^{i}$ so that the closed loop plant is equivalent to the system described by the controller. However, we provide a more convenient way to specify controller stability directly, as described below. Benchmarks assessing the value of a previous version of HIFOO for strong stabilization appear in Gumussoy et al. [2008].

\section{OPTIMIZATION METHOD}

HIFOO 2.0 searches for local minimizers of (1)-(2). The algorithm has two phases. In each phase the main workhorse is the BFGS optimization algorithm, which is surpris- 
ingly effective for nonconvex, nonsmooth optimization, see Lewis and Overton [2009]. The user can provide an initial guess for the desired controller (see below); if this is not provided, HIFOO generates randomly generated initial controllers, and even when an initial guess is provided, HIFOO generates some additional randomly generated initial controllers in case they provide better results.

The first phase is stabilization: BFGS is used to minimize the maximum of the spectral abscissae of the closed loop plants for which $g_{j}$ is either ' $h$ ' or ' $r$ '. This process terminates as soon as a controller is found that stabilizes these plants, thus providing a starting point for which the objective function for the second phase is finite.

The second phase is optimization: BFGS is used to look for a local minimizer of the following unconstrained problem:

$$
\min _{K} F(K)+\rho \sum_{j=1}^{N} \max \left(0, g_{j}(K)-\beta_{j}\right),
$$

where $F$ is the objective function defined in (1) and $\rho$ is a positive penalty parameter multiplying the sum of the constraint violations. If BFGS is unable to find a point for which the constraint violations are zero, the penalty parameter $\rho$ is increased and the optimization is repeated as needed (unless $F$ is identically zero, that is all $\beta_{j}$ are finite). Although there are no guarantees, very often this process is quite effective and reasonably fast. By default, HIFOO invokes the gradient sampling method of Burke et al. [2005] after BFGS terminates, but this is generally more time consuming, and can be avoided as explained in the next section.

\section{USER INTERFACE}

HIFOO is written in MATLAB. The simple call

$$
\mathrm{K}=\operatorname{hifoo}(\mathrm{P} \text {, order })
$$

looks for a controller K solving Scenario 2 above: stabilize the plants described by $\mathrm{P}$ and minimize the sum of the $\mathcal{H}_{\infty}$ norms of the closed loop plants, using a controller of the specified order. Here $\mathrm{P}$ is a cell array of plants specified in any of several formats, typically using the ss class of the MATLAB Control System Toolbox. A useful abbreviation is $P\{j\}=' K$ ', which specifies that the closed loop system for the $j$ th plant is the controller itself. If the order argument is omitted, the default order 0 is used (static output feedback).

A more general calling sequence is

$$
\begin{aligned}
& {[K, F, v i o l]=\text { hifoo }(P, \text { order, init, fun, ... }} \\
& \text { upperbnd, options) }
\end{aligned}
$$

where $\mathrm{P}$ and order are as above, init is an initial guess for the controller (several formats are supported), fun is a string specifying the characters defining the supported functions $g_{j}$ (see Section 2) or a single character if all $g_{j}$ are the same (default: ' $\mathrm{h}$ '), upperbnd is an array specifying the upper bounds $\beta_{j}$ (default: all bounds set to $\infty$ ), and options is a structure with various optional fields, some of which are described below. The order of the input arguments does not matter except that $\mathrm{P}$ must be first. The outputs are, in addition to the controller $K$, the value of the objective function $F(K)$ in (1) and a vector of constraint violations $\max \left(0, g_{j}(K)-\beta_{j}\right)$. There is a fourth output argument loc ("local optimality certificate") that can be requested if gradient sampling is used in addition to BFGS.

Some of the more useful fields in options are:

- options.cpumax: requests HIFOO to quit when the CPU time in seconds exceeds this quantity (default: $\infty)$

- options.fast: 1 to use a fast optimization method only (BFGS), 0 to finish optimization with a slower method (gradient sampling, which may give a better answer) (default: 0, as long as quadprog is in the path; see below)

- options .prtlevel: one of 0 (no printing), 1 (minimal printing), 2 or 3 (more verbose) (default: 1)

- options.struct: specifies sparsity structure to be imposed on the controller (see documentation for details)

- options.weightNormK: weight for adding a penalty on the size of the controller to the objective function, specifically $\left(\left\|A_{K}\right\|^{2}+\left\|B_{K}\right\|^{2}+\left\|C_{K}\right\|^{2}+\left\|D_{K}\right\|^{2}\right)^{1 / 2}$ (default: 0)

- options augmentHinf: weight for adding the reciprocal of the complex stability radius to the $\mathcal{H}_{\infty}$ norm to avoid closed loop plants that are only marginally stabilized: applies to all plants for which $g_{j}$ is ' $\mathrm{h}$ ' (default: 0)

HIFOO 2.0 uses the following external software:

- required: HANSO 1.0, a hybrid algorithm for nonsmooth optimization, freely available from the HIFOO web page;

- required: the matlab Control System Toolbox, for $\mathcal{H}_{\infty}$ norm and complex stability radius computation; this also provides user-friendly system modeling with the ss class;

- optional: quadprog from either the MATLAB Optimization Toolbox or MOSEK; needed only by the gradient sampling part of the algorithm, which is not required.

\section{BENCHMARKS}

We consider a number of simultaneous stabilization problems from the literature; in each case we wish to stabilize multiple plants with a single controller. We consider both Scenario 1 (optimizing the spectral abscissa, by a call such as $K=\operatorname{hifoo}\left(P,{ }^{\prime}, n\right)$, where $n$ is the order of the controller), and Scenario 2 (optimizing $\mathcal{H}_{\infty}$ performance, by a call such as $K=\operatorname{hifoo}(P, ' h$ ', $n)$, or equivalently $\mathrm{K}=\operatorname{hifoo}(\mathrm{P}, \mathrm{n}))$. Notice that we do not need to explicitly set the upper bounds to $\infty$, since that is the default value.

The following MATLAB script illustrates the process, using Scenario 1. There are three first-order plants, given in Jia and Ackermann [2001], and we wish to stabilize them with a first-order controller:

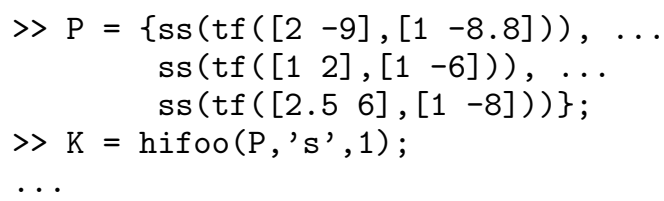


hifoo: best order 1 controller found

has spectral abscissa -0.284216

...

$>\operatorname{tf}(\mathrm{K})$

Transfer function:

$\mathrm{s}+1.182$

$\mathrm{s}+1.595$

$>\operatorname{eig}($ feedback $(\mathrm{P}\{1\},-\mathrm{K}))$

ans $=$

$-0.2842+1.8223 i$

$-0.2842-1.8223 i$

> $\operatorname{eig}($ feedback $(\mathrm{P}\{2\},-\mathrm{K}))$

ans $=$

1. 0 e+009*

$-4.5265$

$-0.0000$

$>\operatorname{eig}($ feedback $(\mathrm{P}\{3\},-\mathrm{K}))$

ans =

$-9.7628$

$-1.4768$

Note that a third-order controller was designed in Jia and Ackermann [2001].

The output of HIFOO may differ on different runs since the initialization is done randomly. However, the output from one run may be used to initialize a second run on the same problem; the result cannot be worse. For example, following the run above with

$\gg \mathrm{K}=\operatorname{hifoo}\left(\mathrm{P}, \mathrm{K}, \mathrm{I}^{\mathrm{S}}\right.$ ', 1$)$;

results in

...

hifoo: best order 1 controller found

has spectral abscissa -0.286524

which is a slight improvement. Depending on the problem and the initial randomization, several successive calls to HIFOO may be required to obtain a stabilizing controller.

We have collected various academic and application examples. We now give benchmark results for Scenarios 1 and 2 .

\subsection{Scenario 1: Simultaneous Stabilization}

To evaluate HIFOO, we consider 31 benchmark problems for simultaneous stabilization as shown in Table 1 . There are 11 problems from applications and 20 academic test problems. The benchmark problems include dynamic, statefeedback and static-output feedback controllers.

Our benchmark results are given in Table 1, showing the problem name, dimensions (with $n^{\max }=\max \left\{n^{i}\right\}$ ), the lowest known controller order from the literature (third column), and the controller orders obtained by HIFOO (final column). For each controller order, HIFOO was run 10 times; the best results are reported. Runs for order $k$ with $k>1$ were initialized with the best controller found for order $k-1$. We used options.fast $=0$ (the default) but all runs were repeated with options. fast $=1$, for which the results were almost the same.

The performance of HIFOO is very good considering the large variety and number of benchmark examples. HIFOO

\begin{tabular}{|c|c|c|c|}
\hline Problem Name & $N \times\left(n^{\max }, m_{2}, p_{2}\right)$ & Known & HIFOO \\
\hline CRJ-200 Aircraft & $6 \times(6,1,6)$ & 0 & 0 \\
\hline F4E Fighter Aircraft & $4 \times(3,1,3)$ & 0 & 0 \\
\hline Gas Turbine Engine & $2 \times(10,2,5)$ & 0 & 0 \\
\hline Helicopter Toy & $4 \times(3,1,1)$ & 2 & 1 \\
\hline Lane-Keeping of AV & $3 \times(2,1,1)$ & 4 & 1 \\
\hline Oblique Wing Aircraft & $64 \times(4,1,1)$ & 0 & 0 \\
\hline PFTC & $6 \times(4,2,4)$ & 0 & 0 \\
\hline RHM14 & $4 \times(8,4,4)$ & 26 & 0 \\
\hline Servomotor & $4 \times(2,1,1)$ & 1 & 1 \\
\hline Ship-Steering & $2 \times(3,1,3)$ & 0 & 0 \\
\hline Stirred-Tank Reactor & $3 \times(2,1,1)$ & 0 & 0 \\
\hline Arehart-Wolovich & $3 \times(2,1,1)$ & 1 & 1 \\
\hline Bhattacharyya et al., Ex. 1-1 & $16 \times(3,1,1)$ & 0 & 0 \\
\hline Bhattacharyya et al., Ex. 1-2 & $16 \times(3,1,1)$ & 1 & 2 \\
\hline Bhattacharyya et al., Ex. 2-1 & $8 \times(2,1,1)$ & 0 & 1 \\
\hline Bhattacharyya et al., Ex. 2-2 & $8 \times(2,1,1)$ & 1 & 1 \\
\hline Blondel et al. & $4 \times(1,1,1)$ & 1 & 0 \\
\hline Bredemann, Ex 4.2 & $3 \times(3,1,1)$ & 2 & 2 \\
\hline Bredemann, Ex 5.5 & $3 \times(2,1,1)$ & 3 & 1 \\
\hline Bredemann, Ex 5.6 & $3 \times(2,1,1)$ & 5 & 0 \\
\hline Bredemann, Ex 5.7 & $3 \times(1,1,1)$ & 1 & 0 \\
\hline Cao-Sun & $3 \times(2,1,1)$ & 0 & 0 \\
\hline Chen et al. & $3 \times(2,3,1)$ & 6 & 0 \\
\hline F.-Anaya et al., Ex.1 & $80 \times(3,1,1)$ & 4 & 0 \\
\hline F.-Anaya et al., Ex.2 & $3 \times(3,1,1)$ & 6 & 1 \\
\hline F.-Anaya et al., Ex.3 & $4 \times(4,1,1)$ & 3 & 1 \\
\hline F.-Anaya et al., Ex.4 & $5 \times(3,1,1)$ & 3 & 0 \\
\hline Gündeş-Kabuli & $5 \times(10,2,2)$ & 4 & 1 \\
\hline Henrion et al., $1^{\text {st }}$ Ex. & $3 \times(1,1,1)$ & 1 & 0 \\
\hline Henrion et al., $2^{\text {nd }}$ Ex. & $3 \times(1,1,1)$ & 1 & 0 \\
\hline Jia-Ackermann & $3 \times(1,1,1)$ & 3 & 1 \\
\hline
\end{tabular}

Table 1. Benchmarks on simultaneous stabilization: 11 industrial and 20 academic examples. The third and fourth columns show the lowest stabilizing controller orders in the literature and the lowest found by HIFOO.

successfully solves the simultaneous stabilization problems with a low-order single controller compared to the existing methods in the literature. Note that contrary to the other methods in the literature, HIFOO allows the user to set the controller order a priori.

In particular, HIFOO is very successful in application problems. HIFOO performs better than existing methods for the application benchmarks Lane-Keeping of Automated Vehicles and Helicopter Toy. The Rationalized Helicopter Model (RHM14) shows the conservativeness of some of the methods in the literature, which produce a 26 th order stabilizing controller. In contrast, HIFOO stabilizes the same benchmark by a static controller.

HIFOO shows similar performance for academic test problems. For almost all problems, the results are better than or equivalent to the results of existing methods. Some of these are Bredemann Ex.5.6, Chen et al., F.-Anaya et al. Ex.1 and Ex.2, and Gündeş-Kabuli, for all of which the existing methods stabilize the benchmark problem with a high-order controller and HIFOO solves the same problem by a static or first-order controller.

There are two benchmark examples for which HIFOO performs slightly worse than the existing methods, namely Ex.1-1 and 2-1 from Bhattacharyya et al. which are interval plants. HIFOO stabilizes both benchmark problems 
with a controller whose order is one more than the order of the controllers in the literature.

\subsection{Scenario 2: Simultaneous $\mathcal{H}_{\infty}$ Optimization}

We start with two academic benchmark problems for simultaneous $\mathcal{H}_{\infty}$ optimization as shown in Table 2 . In the first problem, there are two plants of order two, with optimal full-order $\mathcal{H}_{\infty}$ performance (using different secondorder controllers) equal to 1.290 and 1.245 respectively. HIFOO finds a single first-order controller with the same $\mathcal{H}_{\infty}$ performance (as measured by the maximum of the norms of the two closed loop plants). Also, HIFOo finds a static controller with performance 1.530 . The previously best known $\mathcal{H}_{\infty}$ performance using a single controller was 1.806, using a fourth-order controller.

The second problem consists of three plants of order two with respective optimal $\mathcal{H}_{\infty}$ norms $1.290,1.245$ and 1.038 using full-order controllers. In the literature a 6 th order simultaneously stabilizing controller is known, with $\mathcal{H}_{\infty}$ performance 1.833. HIFOO achieves the optimal $\mathcal{H}_{\infty}$ performance with a single first-order controller.

\begin{tabular}{lccc}
\hline Problem Name & $N \times\left(n^{\max }, m_{2}, p_{2}\right)$ & Known & HIFOO \\
\hline Cao-Lam 1, Ex 2 & $2 \times(2,1,1)$ & $(4,1.806)$ & $(1,1.290)$ \\
& & & $(0,1.530)$ \\
Cao-Lam 2 & $3 \times(2,1,1)$ & $(6,1.833)$ & $(1,1.290)$ \\
& & & $(0,1.530)$ \\
\hline
\end{tabular}

Table 2. Benchmarks on simultaneous $\mathcal{H}_{\infty}$ optimization: two academic examples. The third and fourth columns show the controller order and the minimum value found for the maximum of the $\mathcal{H}_{\infty}$ norms of the closed loop plants, in the literature and by HIFOO.

Table 3 extends the benchmarks for simultaneous stabilization in Table 1 to $\mathcal{H}_{\infty}$ performance optimization. Performance channels are added to each plant in Table 1 using the augw function in the Control System Toolbox by

$\mathrm{G}\{\mathrm{k}\}=\operatorname{augw}(\mathrm{P}\{\mathrm{k}\}, \operatorname{tf}(1,[11]),[], 0.2) ;$

where the weighting functions are $W_{1}(s)=\frac{1}{s+1}$ and $W_{2}(s)=0.2$. The dimensions of the plant $P^{i}$ are $A^{i} \in$ $\mathbb{R}^{n^{i}+1 \times n^{i}+1}, D_{12}^{i} \in \mathbb{R}^{2 p_{2} \times m_{2}}, D_{21}^{i} \in \mathbb{R}^{p_{2} \times m_{2}}$.

These results clearly demonstrate that HIFOO is very effective over various types of benchmark examples including industrial application and academic test problems.

HIFOO 2.0 is available under the GNU Public License at www.cs.nyu.edu/overton/software/hifoo

Further information on references for the benchmark problems and other methods will be provided in a technical report that will be made available at this website.

In conclusion, HIFOO, by allowing the designer to specify the controller order, is a useful tool for simultaneous stabilization and simultaneous $\mathcal{H}_{\infty}$ optimization.

\section{REFERENCES}

Apkarian, P., D. Noll and A. Rondepierre (2008). Mixed $\mathcal{H}_{2} / \mathcal{H}_{\infty}$ control via nonsmooth optimization. SIAM J. Control Optim., 47(3):1516-1546.

\begin{tabular}{|c|c|}
\hline CRJ-200 Aircraft & $(2,2.218),(1,9.333),(0,41.612)$ \\
\hline F4E Fighter Aircraft & $(2,2.993),(1,3.065),(0,6.272)$ \\
\hline Gas Turbine Engine & $(2,1.000),(1,1.000),(0,1.009)$ \\
\hline Helicopter Toy & $(3,0.668),(2,0.845),(1,1.079)$ \\
\hline Lane-Keeping of AV & $(3,0.916),(2,0.947),(1,0.964)$ \\
\hline Oblique Wing Aircraft & $(2,0.488),(1,0.555),(0,0.959)$ \\
\hline PFTC & $(2,1.021),(1,1.022),(0,1.027)$ \\
\hline RHM14 & $(2,0.326),(1,0.374),(0,0.867)$ \\
\hline Servomotor & $(3,0.202),(2,0.202),(1,0.249)$ \\
\hline Ship-Steering & $(2,1.028),(1,1.033),(0,1.041)$ \\
\hline Stirred-Tank Reactor & $(2,6.959),(1,6.968),(0,52.921)$ \\
\hline Arehart-Wolovich & $(3,2.823),(2,2.823),(1,2.917)$ \\
\hline Bhattacharyya et al., Ex. 1-1 & $(2,1.000),(1,1.000),(0,1.000)$ \\
\hline Bhattacharyya et al., Ex. 1-2 & $(4,2.236),(3,2.535),(2,16.441)$ \\
\hline Bhattacharyya et al., Ex. 2-1 & $(3,0.202),(2,0.203),(1,0.204)$ \\
\hline Bhattacharyya et al., Ex. 2-2 & $(3,3.725),(2,3.739),(1,3.745)$ \\
\hline Blondel et al. & $(2,3.053),(1,3.053),(0,3.053)$ \\
\hline Bredemann, Ex 4.2 & $(4,1.623),(3,1.736),(2,1.772)$ \\
\hline Bredemann, Ex 5.5 & $(3,1.515),(2,1.522),(1,1.682)$ \\
\hline Bredemann, Ex 5.6 & $(2,31.613),(1,31.613),(0,31.613)$ \\
\hline Bredemann, Ex 5.7 & $(2,1.073),(1,1.173),(0,7.517)$ \\
\hline Cao-Sun & $(2,0.201),(1,0.202),(0,0.202)$ \\
\hline Chen et al. & $(2,0.595),(1,0.875),(0,1.000)$ \\
\hline F.-Anaya et al., Ex.1 & $(2,0.200),(1,0.200),(0,0.200)$ \\
\hline F.-Anaya et al., Ex.2 & $(3,0.256),(2,0.256),(1,0.256)$ \\
\hline F.-Anaya et al., Ex.3 & $(3,1.000),(2,1.000),(1,1.000)$ \\
\hline F.-Anaya et al., Ex.4 & $(2,0.201),(1,0.203),(0,0.203)$ \\
\hline Gündeş-Kabuli & $(3,1.000),(2,1.000),(1,1.000)$ \\
\hline Henrion et al., $1^{\text {st }}$ Ex. & $(2,0.7155),(1,0.759),(0,1.059)$ \\
\hline Henrion et al., $2^{\text {nd }}$ Ex. & $(2,3.044),(1,3.044),(0,3.455)$ \\
\hline Jia-Ackermann & $(3,2.556),(2,3.080),(1,13.594)$ \\
\hline
\end{tabular}

Table 3. Benchmarks on simultaneous $\mathcal{H}_{\infty}$ optimization: 11 industrial and 20 academic examples. The second column shows the lowest three stabilizing controller orders found by HIFOO and the corresponding maximum of the

$\mathcal{H}_{\infty}$ norms of the closed loop plants.

Blondel, V. D. (1994) Simultaneous stabilization of linear systems. Springer.

Boyd, S. P. and G. H. Barratt (1991). Linear control design: limits of performance. Prentice Hall.

Boyd, S. P., L. El Ghaoui, E. Feron and V. Balakrishnan (1994). Linear matrix inequalities in system and control theory. SIAM, 1994.

Burke, J. V., A. S. Lewis and M. L. Overton (2005). A robust gradient sampling algorithm for nonsmooth, nonconvex optimization. SIAM J. Optimization, 15:751779 .

Burke, J. V., D. Henrion, A. S. Lewis and M. L. Overton (2006). HIFOO - A Matlab package for fixed-order controller design and H-infinity optimization. Proc. IFAC Symp. Robust Control Design, Toulouse, France.

Lewis, A. S. and M. L. Overton (2009). Nonsmooth optimization via BFGS. Submitted to SIAM J. Optimization.

Doyle J., B. Francis and A. Tannenbaum (1990). Feedback Control Theory. Macmillan.

Gumussoy, S., M. Millstone and M. L. Overton (2008). $\mathrm{H}$-infinity strong stabilization via HIFOO, a package for fixed-order controller design. Proc. IEEE Conf. Decision and Control, Cancún, Mexico.

Jia, Y. and J. Ackermann (2001). Condition and algorithm for simultaneous stabilization of linear plants. Automatica 37(9):1425-1434. 
Peaucelle, D. and D. Arzelier (2006). Robust multiobjective control toolbox. Proc. IEEE Symp. Computer-Aided Control System Design, Munich, Germany.

www.laas.fr/OLOCEP/romuloc

Scherer, C. W., P. Gahinet and M. Chilali (1997). Multiobjective output feedback control via LMI optimization. IEEE Trans. Autom. Control, 42(7):896-911.

Vidyasagar M. (1985). Control system synthesis: a factorization approach. MIT Press.

Zhou, K., J. C. Doyle and K. Glover (1996). Robust and optimal control. Prentice Hall. 